\title{
A stone down below: a urethral stone causing acute urinary retention and renal failure
}

\author{
Hanna Bielawska, MD; Norman L. Epstein, MD
}

\section{ABSTRACT}

Impaction of a kidney stone in the male urethra is a rare sequela of an otherwise common disease process. Case reports of urethral stones in the recent literature are scarce. We report a case of a 48-year-old man who presented with an impacted urethral stone as a complication of nephrolithiasis. The pathology was twice missed, even with computed tomography showing the stone in the prostatic urethra, which highlights the challenges of making this diagnosis. We review the existing literature outlining the pathogenesis, clinical features and therapeutic considerations as they relate to urethral stones. We underscore the role of the emergency physician in the diagnosis and initial management of this entity, and draw attention to the need to evaluate not just the upper but also the lower genitourinary tracts when interpreting computed tomographic images obtained for the diagnosis of renal colic.

Keywords: renal colic, urethral stone, computed tomographic urography

\section{RÉSUMÉ}

Un calcul rénal enclavé dans l'urètre chez un homme est une séquelle rare d'un processus morbide généralement courant. On trouve peu de rapports de cas de calculs urétraux dans la récente littérature. Nous présentons le cas d'un homme de 48 ans qui avait un calcul urétral enclavé, comme complication d'une lithiase urinaire. À deux reprises, la pathologie n'a pas été diagnostiquée, même sur des images tomographiques montrant le calcul dans l'urètre prostatique, ce qui met en évidence la difficulté de poser ce diagnostic. Nous avons passé en revue la littérature existante décrivant la pathogenèse, les caractéristiques cliniques et les considérations thérapeutiques des calculs urétraux. Nous soulignons le rôle du médecin d'urgence dans le diagnostic et la prise en charge initiale de cette entité, et attirons l'attention sur la nécessité d'examiner non seulement la partie supérieure du tractus génito-urinaire mais aussi la partie inférieure lors de I'interprétation des images tomographiques obtenues pour le diagnostic de colique néphrétique.

\section{INTRODUCTION}

Urinary tract stones are a common disease entity in industrialized countries, with nephrolithiasis accounting for the majority of clinical presentations. Urethral stones, however, are rare and account for less than $2 \%$ of all urinary tract stones. They most commonly originate from the upper urinary tract or from the bladder, but can form in situ. ${ }^{1-3}$ When a stone occludes the urethra, it can cause acute urinary retention, urethral injury and obstructive renal failure. ${ }^{4}$ Diagnosing a urethral stone is challenging, as symptoms can be nonspecific, and not every imaging modality used in the evaluation of nephrolithiasis includes the lower genitourinary tract. Failure to diagnose an impacted urethral stone can lead to long-term urethral damage, incontinence and renal insufficiency. ${ }^{1-5}$

\section{CASE REPORT}

A 48-year-old man presented to the emergency department (ED) with a 2-day history of suprapubic pain radiating bilaterally to the groin, frequent passage of small volumes of urine, and gross hematuria. He denied fever or back pain. He described the pain as similar in quality to his previous renal colic and noted that he had passed several small stones over the past 2 days. His medical history was otherwise unremarkable and he did not take any medications. On examination, his temperature was $36.3^{\circ} \mathrm{C}$, pulse was 74 beats $/ \mathrm{min}$, respiratory rate was 18 breaths/min and blood pressure was $153 / 100 \mathrm{~mm} \mathrm{Hg}$. The abdominal examination was unremarkable and there were no hernias or costovertebral angle tenderness. Genital and rectal examinations were not performed. Urinalysis revealed greater than 25 red blood cells per highpowered field, 5-10 white blood cells per high-powered

From the Emergency Department, Credit Valley Hospital, Mississauga, Ont.

Submitted Jul. 27, 2009; Revised Nov. 13, 2009; Accepted Dec. 28, 2009

This article has been peer reviewed.

CJEM 2010;12(4):377-80 
field and positive nitrites. A diagnosis of urinary tract infection was made and the patient was discharged with a prescription for ciprofloxacin and phenazopyridine. $\mathrm{He}$ was advised to follow up with his family physician.

The patient returned to the ED the next morning complaining of persistent suprapubic pain, a sensation of pelvic "pressure," penile pain and bilateral flank pain. Despite receiving 2 doses of ciprofloxacin, he continued to experience gross hematuria and frequent passage of small volumes of urine. He was unable to sleep because of pain. He denied fever, nausea, vomiting or passing more stones. On examination, he was in distress. His temperature was $36.4^{\circ} \mathrm{C}$, pulse was 84 beats $/ \mathrm{min}$, respiratory rate was 18 breaths/min and blood pressure was 143/88 mm Hg. The abdominal and costovertebral angle examinations were again benign. Palpation of the testicles and penis failed to reveal any mass or tenderness. A rectal examination was not done. Blood tests revealed a normal complete blood count, normal electrolytes, an elevated creatinine level of $182 \mu \mathrm{mol} / \mathrm{L}$ and a blood urea nitrogen level of $7.8 \mathrm{mmol} / \mathrm{L}$. Urinalysis demonstrated greater than 25 red blood cells per highpowered field and 0-2 white blood cells per high-powered field. Noncontrast computed tomographic (CT) urography was ordered for evaluation of suspected renal colic. The scan was interpreted by the emergency physician as showing bilateral hydronephrosis with small calculi in the distal right ureter, consistent with renal colic (Fig. 1A-C). The patient felt much better after parenteral analgesia and was discharged home with a diagnosis of renal colic. Outpatient urologic follow-up was arranged.

Several hours after the patient was discharged, a verbal report of the CT scan was provided to a second emergency physician by the radiologist on call, indicating there was a $12-\mathrm{mm}$ stone in the proximal urethra just beyond the prostate (Fig. 1D). The patient was called and asked to return to the ED. On his return, he was again in severe discomfort. He received fentanyl and midazolam for analgesia and sedation. Repeat blood tests revealed a creatinine level of $179 \mu \mathrm{mol} / \mathrm{L}$ and a blood urea nitrogen level of $8.7 \mathrm{mmol} / \mathrm{L}$. A portable bladder scan was done and showed $894 \mathrm{~mL}$ of retained urine. Urology was consulted for definitive management. The same evening, the patient underwent urgent cystoscopy with manipulation of the stone from the prostatic urethra into the bladder. $\mathrm{He}$ remained in hospital overnight and was discharged home the next morning with a Foley catheter.

Six weeks later, the patient underwent lithotripsy of what was now a bladder stone, and passed the stone fragments uneventfully. Urine cultures were negative.
The patient's renal function normalized to a creatinine level of $88 \mu \mathrm{mol} / \mathrm{L}$, and he did not sustain any lasting urethral damage.

\section{DISCUSSION}

Urethral stones are a rare occurrence in the industrialized world, accounting for $0.3 \%-2 \%$ of all urinary tract stones. ${ }^{1-3}$ They occur almost exclusively in men, in whom the urethra is longer and more tortuous, although cases in women and children have been reported., ${ }^{4,-8}$ Urethral stones can form in situ secondary to urethral pathology such as strictures and diverticula (primary stones), ${ }^{2,9}$ but more commonly originate from the kidneys or the bladder (secondary stones). ${ }^{5}$ Most urethral stones in the developed world contain calcium oxalate or phosphate, reflecting the composition of renal stones. ${ }^{3,5}$ In developing countries, where bladder stones are far more common (because of a complex interplay of socio-economic and nutritional factors), urethral stones are predominantly composed of struvite or uric acid., ${ }^{1,5,7}$ The impaction of a stone usually occurs at the level of the prostate, although up to $30 \%$ of stones may be found in the anterior urethra. ${ }^{5}$ To cause obstruction, the stones generally have to be larger than $1 \mathrm{~cm}$ in diameter.

The most common presentation of an impacted urethral calculus is acute urinary retention. ${ }^{4,10}$ The stones can also produce irritative and obstructive urinary symptoms, as well as severe pain. ${ }^{1,7}$ The pain of posterior urethral stones is typically referred to the perineum, and patients with anterior stones may present with localized penile pain. If outflow obstruction leads to hydronephrosis, the patient's symptoms may closely mimic the clinical picture of renal colic. Physical examination may reveal costovertebral angle tenderness, a palpable bladder, and, infrequently, the presence of a palpable stone on rectal examination or within the penis. ${ }^{1,3,49}$ The differential diagnosis would include cystitis, pyelonephritis, renal colic, or acute urinary retention secondary to benign prostatic hypertrophy, prostatitis, hemorrhage or a genitourinary malignancy.

Investigations should include routine blood tests and urinalysis, and may reveal renal insufficiency and some degree of hematuria. Urine cultures should be screened to rule out infection. Several imaging modalities, even when ordered for the evaluation of the upper urinary tract, can reveal the presence of a urethral calculus. Because the majority of upper urinary stones are radiopaque, kidney, ureter and bladder films or penile radiographs may be adequate to confirm the diagnosis. 
In one series, $98 \%$ of stones were visible with these simple methods, and the missed diagnoses were attributed to the film ending caudally just above the symphysis pubis. ${ }^{3}$ Ultrasonography can also be used to confirm the presence of urethral calculi, ${ }^{11}$ though penile ultrasonography would need to be specifically requested and is not part of the standard evaluation of renal colic. In addition, bedside emergency ultrasonography can be used to diagnose urinary retention as well as to localize urethral stones in the penile urethra. ${ }^{12}$ Finally, abdominal computed tomography with a renal colic protocol has become
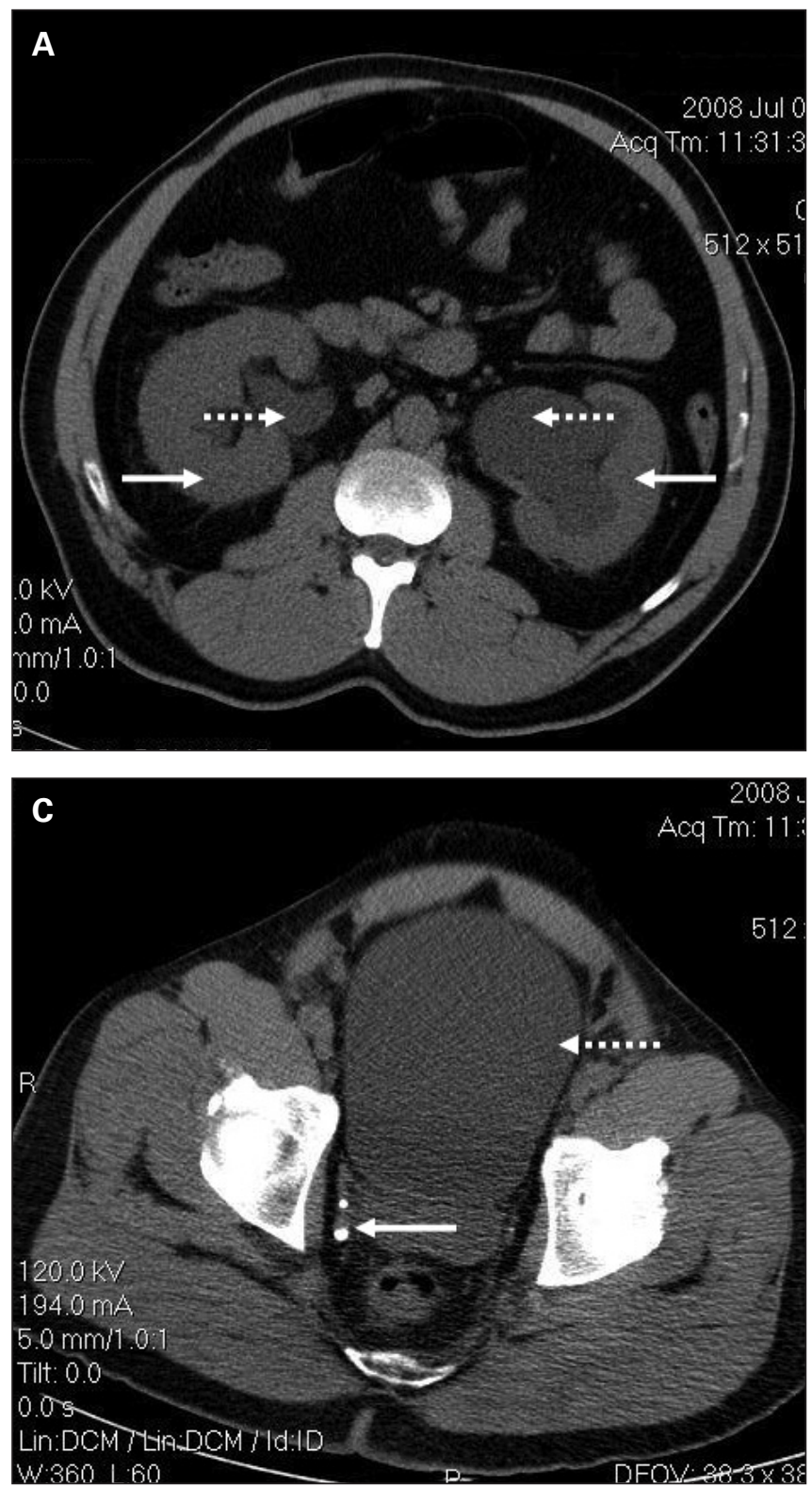

the gold standard for the evaluation of nephrolithiasis, because of its excellent sensitivity and specificity. ${ }^{13}$ There are no reports on the sensitivity of CT urography for the detection of urethral stones, and to our knowledge this is the first report to highlight its utility for this purpose. Since these scans generally include the pelvis and therefore the lower urogenital tract, we reason that urethral stones, when present, should be readily visible on all CT urograms.

Failure to recognize and to remove an obstructing urethral stone can lead to a host of complications, such
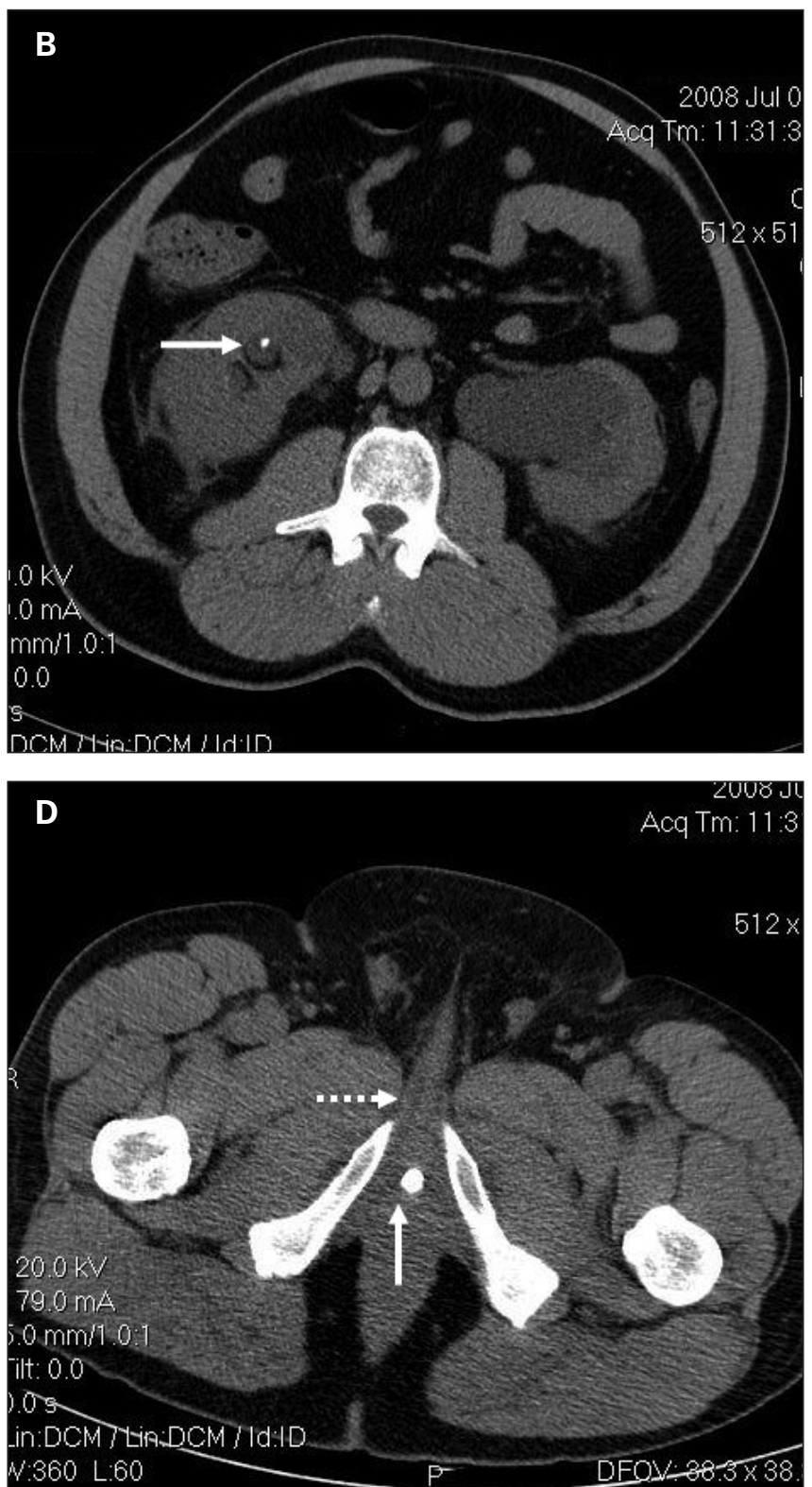

Fig. 1. (A) Computed tomographic scan of the abdomen of a 48-year-old man showing bilateral hydronephrosis (solid arrows) and bilateral hydroureter (dotted arrows). (B) A small nonobstructing right renal stone (arrow), showing evidence of nephrolithiasis. (C) A small nonobstructing calculi at the right ureterovesicular junction (solid arrow) and a distended bladder (dotted arrow). (D) A large calculus in the prostatic urethra (solid arrow), just proximal to the base of the penis (dotted arrow). 
as postobstructive renal failure, long-term urethral damage, urethrocutaneous fistulas, incontinence and impotence. ${ }^{10}$ The objectives of treatment are to provide analgesia, relieve the urinary obstruction and remove the stone without damaging the urethra. The level of involvement of the emergency physician will vary depending on the availability of urologic consultation. Certainly, providing adequate analgesia and antibiotic coverage is imperative. If definitive urologic intervention will be delayed, suprapubic catheterization may be necessary to relieve the outflow obstruction, ${ }^{1,4}$ and can be done under emergency ultrasonographic guidance. ${ }^{14}$ Ultimately, we recommend that stone extraction be carried out under the guidance of a urologist. A urologist's chosen approach will depend on the size and location of the stone, local expertise and presence of urethral pathology. ${ }^{1,9}$ Treatment options include push-back into the bladder for subsequent lithotripsy, in situ lithotripsy and open surgery. ${ }^{1,3,6,15-17}$ Some reports support the judicious extraction of distal stones with forceps or facilitating manual expulsion with instillation of $2 \%$ lidocaine jelly. ${ }^{1}$ Others warn that attempts to milk distal stones are inadvisable, as this is a traumatizing procedure to the delicate urethral epithelium and failed attempts inevitably lead to the formation of permanent urethral fistulas. ${ }^{16,18}$ A recent report by Villaume and colleagues ${ }^{12}$ describes a technique for removing small $(<5 \mathrm{~mm})$ distal urethral calculi under emergency ultrasonographic guidance. However, we recommend expert consultation before an attempt at stone extraction is made.

Though urethral stones are uncommon in clinical practice, in hindsight, the urethral stone in our patient did present in a classic fashion: a prodrome of renal colic, followed by irritative voiding symptoms and acute urinary retention. Fortunately, computed tomography was ordered, and the correct diagnosis was made. These scans are often initially interpreted by emergency physicians, with formal radiologic interpretation arriving sometimes several hours later. We therefore recommend that, in similar settings, clinicians extend the usual practice of looking just at the kidneys and ureters, and include the lower urinary tract as well. Examination of the bladder and the urethra, to look for distension and stones, may at times reveal a surprising complication.

\section{CONCLUSION}

Urethral stones are a rare complication of nephrolithiasis. The diagnosis should be considered in male patients with irritative voiding symptoms or acute urinary reten- tion, especially if there is a history of renal stone disease or symptoms consistent with renal colic. Following an accurate diagnosis, prompt treatment along with urologic consultation is necessary to avoid long-term urologic complications.

Competing interests: None declared.

\section{REFERENCES}

1. Khai-Linh VH, Segura JW. Chapter 84: Lower urinary tract calculi. In: Wein AJ, Kavoussi LR, Novick AC, et al, editors. Campbell's urology. 9th ed. St. Louis (MO): Saunders Elsevier; 2007.

2. Gogus O, Isikay L, Yaman O, et al. An usually large anterior urethral stone. Br J Urol 1995;76:801-2.

3. Verit A, Savas M, Ciftci H, et al. Outcomes of urethral calculi patients in an endemic region and an undiagnosed primary fossa navicularis calculus. Urol Res 2006;34:37-40.

4. Hemal AK, Sharma SK. Male urethral calculi. Urol Int 1991; 46:334-7.

5. Kamal BA, Anikwe RM, Darawani H, et al. Urethral calculi: presentation and management. BJU Int 2004;93:549-52.

6. Durazi MH, Samiei MR. Ultrasonic fragmentation in the treatment of male urethral calculi. Br J Urol 1988;62:443-4.

7. Suzuki $Y$, Ishigooka $M$, Hayami $S$, et al. A case of primary giant calculus in female urethra. Int Urol Nephrol 1997;29:237-9.

8. Thompson B, Hardwood-Nuss AL. Urolithiasis in a child: an uncommon presentation of an uncommon disease. Ann Emerg Med 1995;25:837-40.

9. Ginesin Y, Bolkier M, Nachmias J, et al. Primary giant calculus in urethral diverticulum. Urol Int 1988;43:47-8.

10. Kaplan M, Atakan IH, Kaya E, et al. Giant prostatic urethral calculus associated with urethrocutaneous fistula. Int 7 Urol 2006;13:643-4.

11. Koga S, Shiraishi K, Saito Y, et al. Sonography of urethral calculi. Urol Int 1993;50:203-4.

12. Villaume F IV, Plummer D, Caroon L. Diagnosis and removal of urethral calculi using bedside ultrasound in the emergency department. Acad Emerg Med 2009;16:1031-2 .

13. Sharma A. Unenhanced helical CT in renal colic. Internet Journal Radiology 2005;4:1.

14. Aguilera PA, Choi T, Durham BA. Ultrasound-guided suprapubic cystostomy catheter placement in the emergency department. 7 Emerg Med 2004;26:319-21.

15. El-Sherif AE, Prasad K. Treatment of urethral stones by retrograde manipulation and extracorporeal shock wave lithotripsy. Br J Urol 1995;76:761-4.

16. Dasgupta HK, Gupta VV. Tube-bouginage technic in the management of urethral stone (case reports). J Postgrad Med 1981;27:187-188b.

17. Al-Ansari A, Shamsodini A, Younis N, et al. Extracorporeal shock wave lithotripsy monotherapy for treatment of patients with urethral and bladder stones presenting with acute urinary retention. Urology 2005;66:1169-71.

18. Selli C, Barbagli G, Carini M, et al. Treatment of male urethral calculi. J Urol 1984;132:37-9.

Correspondence to: Dr. Hanna Bielawska, Emergency Department, Credit Valley Hospital, 2200 Eglinton Ave. W., Mississauga ON L5M 2N1; hanna.bielawska@utoronto.ca 